\title{
Treatment outcomes of adjunctive surgery in multidrug-resistant and extensively drug-resistant tuberculosis
}

\section{Opinion}

In 2013, the World Health Organization (WHO) reported that $3.5 \%$ and $20.5 \%$ of new and previously treated tuberculosis (TB) cases were multidrug-resistant tuberculosis (MDR-TB, resistant to both isoniazid and rifampicin), respectively, and $9.0 \%$ of them developed extensively drug-resistant tuberculosis (XDR-TB, resistant to isoniazid, rifampicin, a fluoroquinolone, and 1 or greater injectable agent). The WHO has estimated a global prevalence of 660,000 cases of MDR-TB and 150,000 MDR-TB related deaths annually. A previous multi-country study revealed that among 1,278 MDR-TB cases, around $7 \%$ had XDR-TB. Only 136,000 MDR-TB cases $(45.3 \%)$ among estimated 300,000 MDR-TB cases have been diagnosed and 97,000 cases $(32.3 \%)$ are treated using appropriate regimens based on drug susceptibility testing (DST). In 2013, the treatment success rate of MDR-TB is only $48.0 \%$; around 47,000 cases improved clinically and biologically. Diagnosis and treatment of these cases is largely suboptimal global programmatic capacity. Currently, MDR-TB/XDRTB treatment regimens are lengthy, costly, toxic, and associated with unfavorable treatment outcomes as compared with drug-susceptible TB. The previous largest observational, retrospective, meta-analytic cohort study of about 10,000 MDR-TB cases ( at least 400 cases of them being XDR-TB) demonstrated $62 \%$ of treatment success rate, $7 \%$ of failing or relapsing rate, $17 \%$ of defaulting rate, and $9 \%$ of death rate. Among XDR-TB subgroup, only $40 \%$ reached the treatment success, $22 \%$ failed treatment or relapsed, $16 \%$ defaulted, and $15 \%$ died. Among the subgroup of XDR-TB with severe drug-resistance pattern, only $19 \%$ achieved treatment success. Thoracic surgery for MDR-TB/XDR-TB is a potentially beneficial adjunctive treatment. The first surgical intervention on a patient with TB was performed by Barry E in 1726, followed by Forlannini C in 1882, who carried out an artificial pneumothorax. A favorable outcomes for 72 MDR-TB/ XDR-TB patients undergoing surgical lung resection in the country of Georgia was reported that $49(68 \%)$ were cured, 6(8\%) were completed. Nevertheless, $4(5.5 \%)$ of them were treatment failure, 5(7\%) were defaulters, and $4(5.5 \%)$ were dead. The surgical procedures were performed as the following : $11 \%$ of pneumonectomy, $54 \%$ of lobectomy, and $35 \%$ of segmentectomy. With utilizing a combination of personal medical treatment for MDR-TB/XDR-TB according to the WHO guidelines and adjunctive surgical treatment, a high rate of favorable treatment outcomes $(82 \%)$ was obtained. Favorable treatment outcomes was included in $90 \%$ of those with MDR-TB and $67 \%$ of those with XDR-TB. Surgical treatment for drug-resistant TB has been demonstrated to be safe and effective, with operative mortality rates similar to surgery for lung cancer. Two specific indications for lung resection in drug-resistant TB are failed medical treatment with persistent sputum positivity and patients with medical treatment and negative sputum smear who have bronchiectasis or localized cavitary disease. A systematic review and meta-analysis of
Volume 5 Issue 2 - 2017

\author{
Attapon Cheepsattayakorn, ${ }^{1,2}$ Ruangrong \\ Cheepsattayakorn ${ }^{3}$ \\ ' IOth Zonal Tuberculosis and Chest Disease Center, Chiang Mai, \\ Thailand \\ ${ }^{2}$ Department of Disease Control, Ministry of Public Health, \\ Thailand \\ ${ }^{3}$ Department of Pathology, Faculty of Medicine, Chiang Mai \\ University, Thailand
}

Correspondence: Attapon Cheepsattayakorn, I0th Zonal Tuberculosis and Chest Disease Center, I 43 Sridornchai Road Changklan Muang Chiang Mai 50100 Thailand, Tel 66-531-40767, 66 5-327-636-4, Fax 665-3|4-077-3, 665-327-359-0, Email Attapon 1958@gmail.com; attaponche@yahoo.com

Received:September 18, 2017 | Published: December 28, 2017

adjunctive pulmonary resection for MDR-TB patients that included 15 studies (a total sample size of 949) demonstrated the overall cure rate of $84 \%$. A previous study of 5 Japanese XDR-TB cases revealed that two cases with pneumonectomy and three cases with upper lobectomy (preoperative chemotherapy with sparfloxacin + pyrazinamide + cyclos erine+ethionamide+enviomycin;gatifloxacin+cycloserine+enviomyci $\mathrm{n}+$ para-aminosalicylic acid; gatifloxacin+pyrazinamide+cycoserine ; gatifloxacin+pyrazinamide+enviomycin ; and kanamycin+cycoserin e+ethionamide+para-aminosalicylic acid+sultamicillin tosilate) After the surgical operation, most patients attained sputum negative status, and return to their normal daily activities. The duration of postoperative antituberculous chemotherapy ranged from 12-25months with the median of 19 months and all of them remained free from disease at the time of follow-up. In conclusion, There has been lack of randomized controlled trials of surgical resections although observational studies with surgical interventions have demonstrated a high treatment success rate when used as adjunctive treatment in MDR-TB/XDR-TB patients. Adjunctive surgical interventions may [lay a significant role in improving clinical outcomes in many cases of complicated MDRTB/XDR-TB with localized and tissue destruction accompanying failure to become culture negative.

\section{Acknowledgements}

None.

\section{Conflict of interest}

The author declares no conflict of interest. 